\title{
The Neutrino Contribution of Gamma-Ray Flares from Fermi Bright Blazars
}

\author{
Kenji Yoshida, ${ }^{a, *}$ Maria Petropoulou, ${ }^{b}$ Kohta Murase ${ }^{c}$ and Foteini Oikonomou ${ }^{d}$ \\ ${ }^{a}$ Shibaura Institute of Technology, \\ 307 Fukasaku, Minuma-ku, Saitama, Japan \\ ${ }^{b}$ National and Kapodistrian University of Athens, \\ 157 72, Athens, Greece \\ ${ }^{c}$ The Pennsylvania State University, \\ University Park, PA, USA \\ ${ }^{d}$ Norwegian University of Science and Technology, \\ 7491, Trondheim, Norway \\ E-mail: yoshida@shibaura-it.ac.jp
}

High-energy neutrinos are expected to be produced during gamma-ray flares of blazars through the interaction of high-energy cosmic rays in the jet with photons. As a matter of fact, a highenergy neutrino event, IC-170922A, was detected at the time of a gamma-ray flare from blazar TXS 0506+056 at the level of 3 sigma significance. In this work, we present a statistical study of blazar gamma-ray flares aiming to constrain their contribution to the blazar neutrino output. We selected 145 gamma-ray bright blazars listed in the Fermi Large Area Telescope (LAT) monitored list and constructed their weekly binned light curves. Using a Bayesian Blocks algorithm to the light curves, we determined the fraction of time spent in the flaring state (flare duty cycle) and the fraction of energy released during each flare. Furthermore, we estimated the neutrino energy flux of each gamma-ray flare by using the general scaling relation $L_{\gamma} \propto\left(L_{\gamma}\right)^{\gamma}, \gamma=1.0-2.0$, normalized to the quiescent X-ray or gamma-ray flux of each blazar. Comparison of the estimated neutrino energy flux with the declination-dependent IceCube sensitivity enables us to constrain the standard neutrino emission models of gamma-ray flares. We also provide the upper-limit contribution of flares of gamma-ray bright blazars to the isotropic diffuse neutrino flux.

$37^{\text {th }}$ International Cosmic Ray Conference (ICRC 2021)

July 12th - 23rd, 2021

Online - Berlin, Germany

\footnotetext{
${ }^{*}$ Presenter
} 


\section{Introduction}

The IceCube Collaboration found high-energy astrophysical neutrinos with an isotropic arrival distribution in the $100 \mathrm{TeV}-\mathrm{PeV}$ range. The observed astrophysical muon neutrino flux from the northern sky with IceCube is represented by $E_{v}^{2} \Phi_{v}(E)=1.0 \times 10^{-8} \mathrm{GeV} \mathrm{cm}^{-2} \mathrm{~s}^{-1} \mathrm{sr}^{-1}$ [1]. The observed diffuse neutrino flux can be regarded as an isotropic neutrino background produced by a large number of extra-galactic sources (e.g. [2]). The IceCube Collaboration et al. (2018) reported a sub-PeV muon neutrino event in 2017, IceCube-170922A, which was coincident in direction and time with a gamma-ray flare of blazar TXS 0506+056 at the statistical significance level of $3 \sigma$ [3]. Independent of and prior to the 2017 flaring event, IceCube Collaboration (2018) also reported $3.5 \sigma$ evidence for neutrino events from the direction of TXS 0506+056 with an archival search of past IceCube data [4].

Blazars are the most extreme subclass of active galactic nuclei (AGNs), characterized by their luminous core, very rapid variability, high polarization, superluminal motion, and broadband nonthermal spectra. It is believed that their relativistic jets from the central supper-massive black hole are oriented close to the observer's line of sight [5]. Blazars are the major candidates of the sources of ultrahigh-energy cosmic rays and high-energy neutrinos (e.g. [6, and references therein]). Blazars are known to be flaring sources, namely they exhibit flux changes of a few orders detected across the electro-magnetic spectrum on different timescales. While the origin of blazar flares remains still a mystery, neutrino production is expected to be enhanced during these high-flux states [e.g., 2].

In this work, we estimate the contribution of gamma-ray flares to the neutrino signal from blazars. Using a sample of 145 gamma-ray blazars, we present the results that constrain the standard leptonic scenario for the blazar gamma-ray emission by comparing with the IceCube sensitivity, and provide the upper-limit contribution of gamma-ray flares of bright blazars to the isotropic diffuse neutrino flux.

\section{Gamma-ray data and analysis}

\subsection{Gamma-ray data}

We selected 145 gamma-ray bright blazars listed in the Fermi Large Area Telescope (LAT) Monitored Source List ${ }^{1}$ with TXS 0506+056. The sample consists of 105 Flat Spectrum Radio Quasars (FSRQs), 31 BL Lacs, and 9 blazar candidates of uncertain type (BCUs). In this work, we analyze Fermi-LAT data from MJD 54682 to MJD 58739 using the Fermi Science Tools version 11-05-032 and Fermipy version 0.17.3 [7]. Gamma-ray photons are selected in the energy range between $100 \mathrm{MeV}$ and $316 \mathrm{GeV}$, retaining only events that arrived at a zenith angle less than $90^{\circ}$ to reduce the Earth-limb gamma-ray contamination. Isotropic and Galactic diffuse gamma-ray backgrounds were included using the parameterization provided in the iso_P8R3_SOURCE_V2_v01.txt and gll_iem_v07.fits files, leaving their all parameters free in the spectral fit. Sources in the 4FGL catalog within a radius of $15^{\circ}$ from the source position were included in the model with their spectral parameters fixed to their catalog values [8], while the normalizations of sources within

${ }^{1}$ https://fermi.gsfc.nasa.gov/ssc/data/access/lat/msl_lc/

2http://fermi.gsfc.nasa.gov/ssc/data/analysis/software 


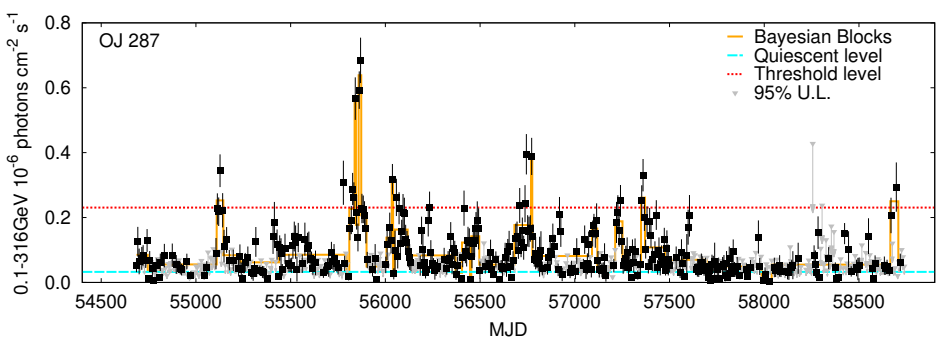

Figure 1: An example of weeklybinned blazar gamma-ray light curves (black points) in the $0.1-$ $316 \mathrm{GeV}$ energy range with $95 \%$ upper limits (grey triangles). The quiescent and flare threshold $(6 \sigma)$ levels are plotted with dashed cyan and red lines, respectively. The Bayesian Blocks representation is over plotted with orange solid lines.

$3^{\circ}$ were allowed to vary freely during the spectral fit. The spectral fit was performed using the P8R3_SOURCE_V2 instrument response function.

The gamma-ray light curve of each source was obtained with an end-to-end analysis in each time bin using the same processing steps as the spectral analysis. The period between MJD 54682 and MJD 58739 was divided into $\sim 570$ of 7 day-long bins. The spectral model of the target source is taken from 4FGL with the all parameters free to vary in the spectral fit. ${ }^{3}$ The best-fit parameters were then used to calculate the photon flux in the $0.1-316 \mathrm{GeV}$ energy range for each time bin of the light curve. Figure 1 shows an example of the gamma-ray light curves. The flux points are shown with their $1 \sigma$ uncertainties (black symbols), if the source is detected with a test statistic (TS) larger than 9 , corresponding to a $3 \sigma$ excess, and the flux in one bin larger than its uncertainty. Otherwise, the flux upper limits are shown at the $95 \%$ confidence level.

\subsection{Definition of flares}

Although there is no rigid way to define the quiescent flux level of blazar emission, it is valid to suppose that the quiescent state has a relatively low and stable flux. In this work, the following procedure is taken to compute the quiescent level:

1. A Bayesian Blocks algorithm is applied to each gamma-ray light curve with the false alarm probability $p_{0}=0.05$ [9-11]. The Bayesian Blocks algorithm provides the partitions of the light curve into the blocks, in which the flux is regarded as a constant flux.

2. The average flux of each Bayesian block is computed as the constant flux of the block.

3. The minimum average flux is take as the quiescent level under the condition that the number of data points of the block is more than or equal to the average number of data points of the blocks, preventing from using an insufficient amount of data points.

As an indicative example, figure 1 shows the weekly-binned Fermi-LAT light curve of OJ 287 (black points) and the Bayesian Blocks representation (solid orange lines). The quiescent flux level computed with the method described above is also indicated with a dashed cyan line.

Here, we define a gamma-ray flare flux as the flux of the weekly-binned light curves that exceeds a certain threshold, $F_{\gamma}^{t h}$, given by

$$
F_{\gamma}^{t h}=F_{\gamma}^{q}+s\left\langle F_{\gamma}^{e r r}\right\rangle
$$

\footnotetext{
${ }^{3}$ e.g. LogParabola with the free parameters of norm, $\alpha$, and $\beta$.
} 

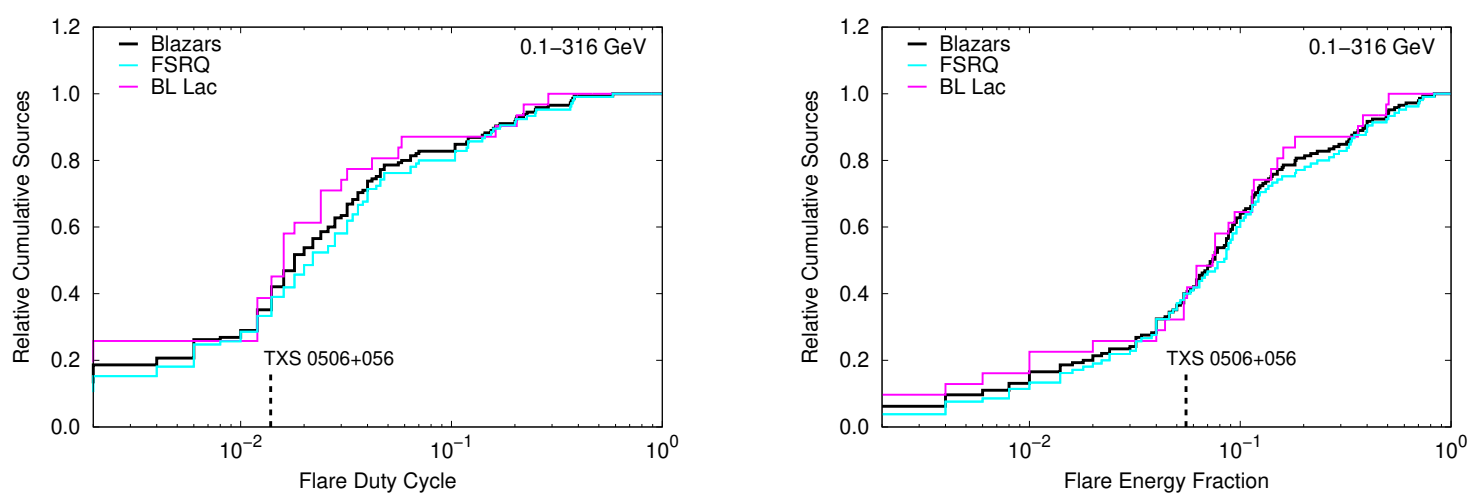

Figure 2: Cumulative histograms of the $0.1-316 \mathrm{GeV}$ gamma-ray flare duty cycles (left panel) and flare energy fractions (right panel) of 145 blazars (black solid), 105 FSRQs (cyan solid), and 31 BL Lacs (magenta solid). In both panels, the vertical dashed line indicates the values of TXS 0506+056

where $F_{\gamma}^{q}$ is the gamma-ray quiescent level, $\left\langle F_{\gamma}^{\text {err }}\right\rangle$ is the average of the gamma-ray flux errors, and $s$ corresponds to the significance above the quiescent level in units of the standard deviation $\sigma$. Unless otherwise noted, we use $s=6$ in this paper. Figure 1 also shows an example of the flaring threshold level of the gamma-ray light curve with a dashed red line.

\subsection{Flare Duty Cycle and Flare Energy Fraction}

The flare duty cycle, that is the fraction of time spent in the flaring state, is given by

$$
f_{f l}=\frac{1}{T_{t o t}} \int_{F_{\gamma}^{t h}} d F_{\gamma} \frac{d T}{d F_{\gamma}}
$$

where $T_{t o t}$ is the total observation time. The gamma-ray flare energy fraction, that is the fraction of energy emitted in the flaring state, is given by

$$
b_{f l}^{\gamma}=\frac{1}{F_{\gamma}^{a v e} T_{t o t}} \int_{F_{\gamma}^{t h}} d F_{\gamma} F_{\gamma} \frac{d T}{d F_{\gamma}}
$$

where $F_{\gamma}^{a v e}$ is the average gamma-ray flux over the whole observation period. Figure 2 presents the cumulative distributions of the flare duty cycles $\left(f_{f l}\right)$ and flare energy fractions $\left(b_{f l}^{\gamma}\right)$ for the $0.1-316 \mathrm{GeV}$ gamma-ray light curves of 145 blazars. The $f_{f l}$ and $b_{f l}^{\gamma}$ values of TXS 0506+056 are indicated with vertical dashed lines. Their differential distributions are well represented by a power-law function with the index of $-1.57 \pm 0.08$ for $f_{f l}>0.01$ and $-1.36 \pm 0.12$ for $b_{f l}^{\gamma}>0.03$, respectively. A two-sample Kolmogorov-Smirnov test shows no significant differences between FSRQs and BL Lacs for the cumulative distributions of $f_{f l}$ and $b_{f l}^{\gamma}$ at the significance level of $10 \%$.

\section{Implication for high-energy neutrino emission}

\subsection{Estimation of Muon Neutrino Flux}

The standard leptonic models of the blazar gamma-ray emission suggest that flares can dominate the neutrino output of a blazar [e.g., 2]. In general, in the leptonic models the neutrino flux is related 


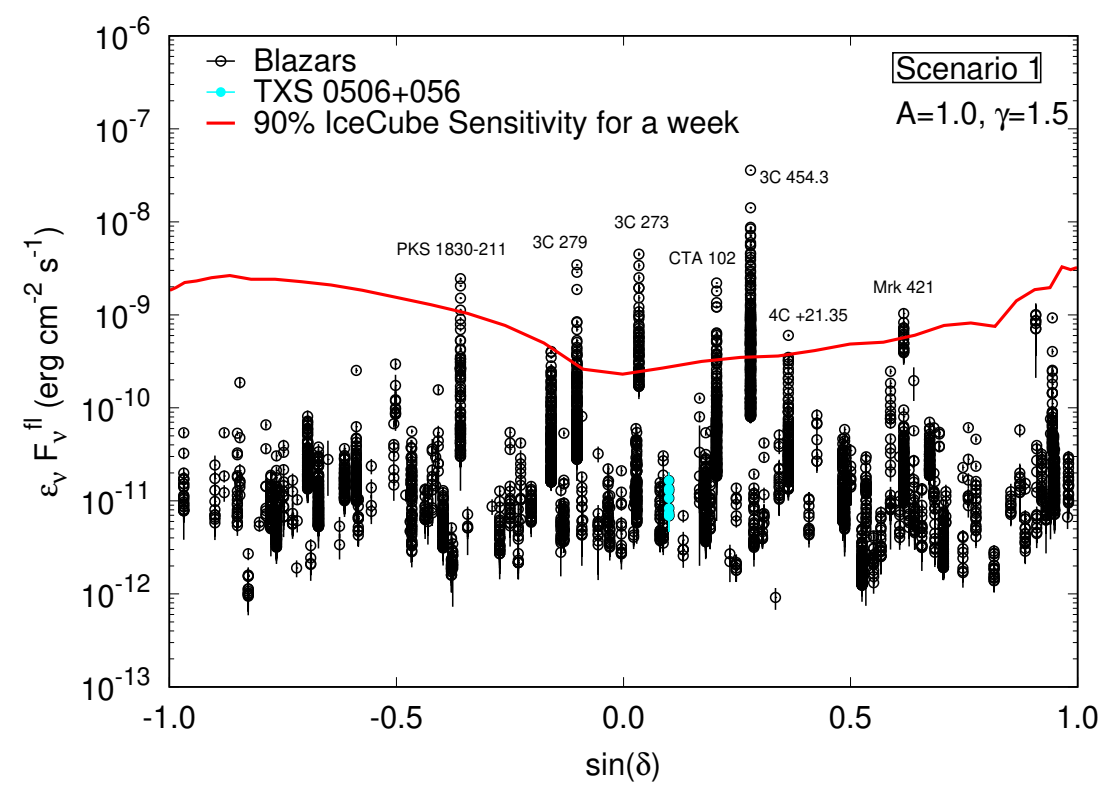

Figure 3: Estimated muon neutrino flare fluxes of scenario 1 with $A=1.0$ and $\gamma=1.5$ as a function of $\sin \delta$. The IceCube $90 \%$ sensitivity is also plotted (red solid line).

to the gamma-ray flux as:

$$
\varepsilon_{\nu} F_{v}^{f l}=\varepsilon_{\nu} F_{v}^{q}\left(\frac{F_{\gamma}^{f l}}{F_{\gamma}^{q}}\right)^{\gamma},
$$

where $\varepsilon_{v} F_{v}^{q}$ is the quiescent neutrino flux, $F_{\gamma}^{f l}$ is the gamma-ray photon flux in the flaring state, and $\gamma$ is the index in the range of $\sim 1.0-2.0$ [2, and references therein]. The quiescent neutrino flux is bench-marked with the following two scenarios.

\subsubsection{Scenario 1: the quiescent $\mathrm{X}$-ray flux}

Motivated by results after TXS 0506+056, the X-ray quiescent flux level in $0.3-10 \mathrm{keV}$ band indicates an upper limit to the muon neutrino quiescent flux in the $100 \mathrm{TeV}-\mathrm{PeV}$ range (for details, see [12, and references therein]). For the X-ray data, we use the Open Universe for Blazars, which provides blazar X-ray light curves based on 14 years of Swift-XRT data [13]. The X-ray quiescent flux level is computed in the same way as the gamma-ray quiescent flux level (see section 2.2). The muon neutrino flare flux, $\varepsilon_{\nu} F_{v_{\mu}}^{f l}$, is then estimated as

$$
\varepsilon_{\nu} F_{\gamma_{\mu}}^{f l}=A \varepsilon_{X} F_{X}^{q}\left(\frac{F_{\gamma}^{f l}}{F_{\gamma}^{q}}\right)^{\gamma}
$$

where $\varepsilon_{X} F_{X}^{q}$ is the $\mathrm{X}$-ray quiescent level and $A$ is a normalization parameter.

We derived the neutrino flare fluxes of 129 blazars (96 FSRQs, 28 BL Lacs, 5 BCUs) using equation (5) and ignoring 16 sources of the non-flaring state with the $6 \sigma$ threshold or the unavailable quiescent state with the Swift X-ray data. Figure 3 shows the estimated muon neutrino flare fluxes from scenario 1 with $A=1.0$ and $\gamma=1.5$ as a function of the $\sin \delta$ ( $\delta$ : declination). The red 
line shows the weekly IceCube $90 \%$ sensitivity calculated with an $E^{-2}$ neutrino spectrum and no background in the energy range $10 \mathrm{GeV}-10 \mathrm{EeV}$. Some blazars such as 3C 273, CTA 102, 3C 454.3, and Mrk 421 exceed the IceCube $90 \%$ sensitivity in the neutrino flare flux.

\subsubsection{Scenario 2: the quiescent $\gamma$-ray flux}

The scenario 2 is motivated by results prior to TXS 0506+056. The quiescent neutrino flux is estimated by the quiescent $\gamma$-ray flux, $\varepsilon_{\gamma} F_{\gamma}^{q}$, at the pivot energies. These correspond to the de-correlation energy to minimize the correlation of the fitted spectral parameters for every weekly bin. Then, the muon neutrino flare flux is represented by

$$
\varepsilon_{\nu} F_{v_{\mu}}^{f l}=A \varepsilon_{\gamma} F_{\gamma}^{q}\left(\frac{F_{\gamma}^{f l}}{F_{\gamma}^{q}}\right)^{\gamma}
$$

\subsection{Contribution of Blazar Flares to the Isotropic Diffuse Neutrino Flux}

Following Murase et al. (2018) [2], in particular their eq. (7), we estimate the contribution of blazar flares to the isotropic diffuse neutrino flux. Setting the solid angle covered by the IceCube detector of $\Delta \Omega=2 \pi$, we adopt the IceCube typical $5 \sigma$ discovery potential for a point source with an $E_{v}^{-2}$ neutrino spectrum of $1.0 \times 10^{-12} \mathrm{TeV} \mathrm{cm}^{-2} \mathrm{~s}^{-1}$ for $8 \mathrm{yr}$, after scaling down to a week becomes $F_{\text {lim }}=6.7 \times 10^{-10} \mathrm{erg} \mathrm{cm}^{-2} \mathrm{~s}^{-1}$. We also take on a luminosity-dependent correction factor determined by the redshift evolution $q_{L}=1$ and a factor of the multiplet analysis $b_{m}=6.6$ for $m \geq 2$. As a result, the contribution of blazar flares to the isotropic diffuse neutrino flux is constrained at the level of

$$
E_{v}^{2} \Phi_{\nu} \lesssim 3.8 \times 10^{-10} \mathrm{GeVcm}^{-2} \mathrm{~s}^{-1} \mathrm{sr}^{-1}\left(\frac{\xi_{z}}{0.7}\right)\left(\frac{0.02}{f_{f l}}\right)^{1 / 2}\left(\frac{b_{f l}^{v}}{0.4}\right)^{1 / 2}\left(\frac{10^{46} \mathrm{ergs}^{-1}}{\varepsilon_{v} L_{\varepsilon_{\nu \mu}}^{f l}}\right)^{1 / 2},
$$

where $\xi_{z}$ is a dimensionless parameter that depends on the redshift evolution of the sources: $\xi_{z}=0.68$ for the gamma-ray luminosity density evolution of BL Lacs and $\xi_{z}=8.4$ for that of FSRQs [14, and references therein]. The neutrino flare energy fraction, $b_{f l}^{v}$, is derived for each source by using the neutrino quiescent flux, flare duty cycle, and neutrino flare fluxes. The neutrino flare luminosities are computed with the redshifts of the blazars [15, 16]: $\varepsilon_{v} L_{v}^{f l}=4 \pi d_{L}^{2} \varepsilon_{v} F_{v}^{f l}$, where $d_{L}$ is a luminosity distance with $\Omega_{m 0}=0.308, \Omega_{\Lambda 0}=0.692$, and $H_{0}=67.8 \mathrm{~km} \mathrm{~s}^{-1} \mathrm{Mpc}^{-1}$ [17].

As shown in Fig. 3 some neutrino flare fluxes with $A=1.0$ are beyond the IceCube $90 \%$ sensitivity. The normalization parameter $A$ is set to 1.0 for the sources whose all flares are less than the sensitivity, while if at least one flare flux of a source exceeds the sensitivity, $A$ of the source is reduced for the maximum flux of the source to be the sensitivity flux. This processing might give the overestimate of the neutrino fluxes under the constrain of the IceCube $90 \%$ sensitivity. The contribution of flares from bright gamma-ray blazars to the isotropic diffuse neutrino flux can be estimated to be the average of the upper-limit values of eq.(7) over all flares.

Figure 4 presents the upper limits of the blazar neutrinos for the isotropic diffuse neutrino flux as a function of the flare significance $s$ of the scenario 1 (solid squares) and scenario 2 (open squares) with $\gamma=1.0$ (blue), $\gamma=1.5$ (dark red), and $\gamma=2.0$ (green). The dashed horizontal 


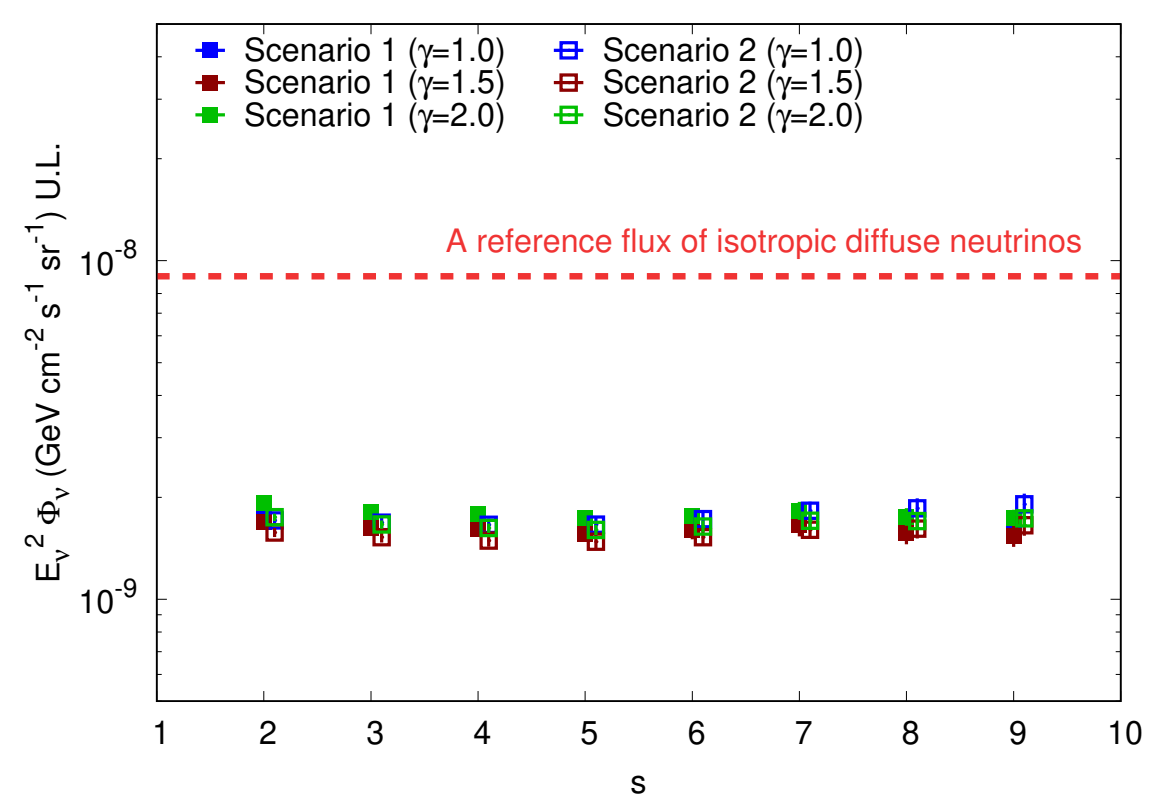

Figure 4: The upper limits of the blazar neutrinos for the isotropic diffuse neutrino flux as a function of the significance $s$ of the scenario 1 (solid squares) and scenario 2 (open squares) with $\gamma=1.0$ (blue), 1.5 (dark red), and 2.0 (green). The dashed red line indicates a reference flux of the isotropic diffuse neutrinos [18].

line indicates a reference flux of the isotropic diffuse neutrinos [18]. The upper limits are almost independent of the significance $s$, the power index $\gamma$, and the two scenarios.

\section{Summary}

From flares of bright gamma-ray blazars, independent of the detailed calculation of neutrino generation, we estimated the neutrino energy flux of each gamma-ray flare by using the simple scaling relation $L_{\gamma} \propto\left(L_{\gamma}\right)^{\gamma}(\gamma=1.0-2.0)$, normalized to the quiescent X-ray and gamma-ray flux of each blazar. Comparison of the estimated neutrino energy flux with IceCube $90 \%$ Sensitivity suggests that the quiescent neutrino fluxes tend to be smaller than the quiescent X-ray and gammaray fluxes, and the power index $\gamma$ tends to be closer to 1.0 rather than 2.0. We also obtained the upper limit of $\sim 20 \%$ of the contribution of bright gamma-ray and neutrino blazars to the isotropic diffuse neutrino flux, following the stacking/clustering constraints derived in $[2$, and references therein]. It should be noted that the dimmer neutrino blazars could make a larger contribution to the isotropic diffuse neutrino flux.

\section{References}

[1] M.G.Aartsen et al., Evidence for Astrophysical Muon Neutrinos from the Northern Sky with IceCube, Phys. Rev. Lett. 115 (2015) 081102 [astro-ph. HE/1507.04005].

[2] K. Murase, F. Oikonomou, \& M. Petropoulou, Blazar Flares as an Origin of High-energy Cosmic Neutrinos?, Astrophys. J. 865 (2018) 124 [astro-ph.HE/1807. 04748]. 
[3] The IceCube Collaboration et al., Multimessenger observations of a flaring blazar coincident with high-energy neutrino IceCube-170922A, Science 361 (2018) eaat1378 [astro-ph.HE/1807.08816].

[4] The IceCube Collaboration, Neutrino emission from the direction of the blazar TXS 0506+056 prior to the IceCube-170922A alert, Science 361 (2018) 147 [astro-ph.HE/1807.0879].

[5] C.M. Urry \& P. Padovani, Unified Schemes for Radio-Loud Active Galactic Nuclei, PASP 107 (1995) 803 [astro-ph/9506063].

[6] K. Murase, Active Galactic Nuclei as High-Energy Neutrino Sources, Neutrino Astronomy: Current Status, Future Prospects (2017) 15 [astro-ph.HE/1511.01590v2].

[7] M. Wood et al., Fermipy: An open-source Python package for analysis of FermiLAT Data, 35th International Cosmic Ray Conference (ICRC2017) 301 (2017) 824 [astro-ph.HE/1707.09551].

[8] S. Abdollahi et al., Fermi Large Area Telescope Fourth Source Catalog, Astrophys. J. Suppl. 247 (2020) 33 [astro-ph. IM/1902 . 10045].

[9] J.D. Scargle et al., Studies in Astronomical Time Series Analysis. VI. Bayesian Block Representation, Astrophys. J. 764 (2013) 167 [astro-ph. IM/1207. 5578].

[10] Astropy Collaboration, Astropy: A community Python package for astronomy, Astron. \& Astrophys. 558 (2013) A33 [astro-ph. IM/1307.6212].

[11] The Astropy Collaboration, The Astropy Project: Building an Open-science Project and Status of the v2.0 Core Package, Astron. J. 156 (2018) 123 [astro-ph. IM/1801.02634].

[12] P. Padovani et al., TXS 0506+056, the first cosmic neutrino source, is not a BL Lac MNRAS 484 (2019) L104 [astro-ph.HE/1901. 06998].

[13] P. Giommi et al., Open Universe for Blazars: a new generation of astronomical products based on 14 years of Swift-XRT data Astron. \& Astrophys. 631 (2019) A116 [astro-ph.HE/1904.06043].

[14] K. Murase \& E. Waxman, Constraining high-energy cosmic neutrino sources: Implications and prospect, Phys. Rev. D 94 (2016) 103006 [astro-ph.HE/1607.01601].

[15] M. Ackermann et al., The Third Catalog of Active Galactic Nuclei Detected by the Fermi Large Area Telescope, Astrophys. J. 810 (2018) 14 [astro-ph.HE/1501.06054].

[16] A. Oberto et al., What is in SIMBAD?, Astronomical Society of the Pacific Conference Series $521(2019) 96$

[17] M. Tanabashi et al., Review of Particle Physics, Phys. Rev. D 98 (2018) 030001

[18] M.G. Aartsen et al., Observation and Characterization of a Cosmic Muon Neutrino Flux from the Northern Hemisphere using six years of IceCube data, Astrophys. J. 833 (2016) 3 [astro-ph.HE/1607.08006]. 